\title{
La imagen del magnetismo animal en la literatura de ficción: los casos de Poe, Doyle y Du Maurier
}

\author{
Juan Marcos Bonet Safont (*) \\ $\left(^{*}\right) \quad$ Instituto de Historia de la Medicina y de la Ciencia «López Piñero», Universidad de \\ Valencia-CSIC. bonetsafont@gmail.com
}

Dynamis

[0211-9536] 2014; 34 (2): 403-423

http://dx.doi.org/10.4321/S0211-95362014000200007
Fecha de recepción: 13 de mayo de 2013

Fecha de aceptación: 21 de noviembre de 2013

SUMARIO: 1.-Introducción. 2.-La verdad sobre el caso del señor Valdemar, Edgar Allan Poe. 3.-El gran experimento de Keinplatz, Conan Doyle. 4.-Trilby, Du Maurier. 5.-Conclusiones.

RESUMEN: En el presente trabajo nos acercamos a la imagen social del fenómeno conocido como mesmerismo o magnetismo animal a través del análisis de las obras: The Facts in the Case of Mr. Valdemar (1845) de Edgar Allan Poe, The Great Keinplatz Experiment (1885) de Conan Doyle y Trilby (1894) de George Du Maurier. Mostraremos cuál es el estereotipo del magnetizador y los usos que observamos del mesmerismo. Nos acercaremos a los espacios y actores del trasunto mesmerico presentado en los relatos. Tendremos en cuenta la recepción por parte del público de estas historias y las relaciones con los conocimientos mesmericos e hipnóticos que tenían los autores de éstas. En la actualidad, investigadores académicos, dentro de la disciplina de la psicología, publican artículos y libros sobre los mitos populares de la hipnosis intentando poner de manifiesto las imágenes distorsionadas referentes a este fenómeno. Esta imagen distorsionada del proceso hipnótico, y del hipnotizador, proviene de los espectáculos circenses de hipnosis (stage hypnosis), del cine, de la televisión y de la literatura de ficción. Por otro lado, tenemos en la literatura de ficción una fuente única e inestimable de datos, ideas, especulaciones, preocupaciones y posibilidades en torno al magnetismo animal e hipnosis que convierten su estudio y análisis en un capítulo imprescindible de cualquier trabajo histórico de este tema. Veremos cómo el uso literario del mesmerismo en el caso de Poe, Doyle y Du Maurier no es algo casual o periférico, sino que todos ellos estuvieron intelectualmente interesados y estimulados por estas ideas.

PALABRAS CLAVE: mesmerismo, magnetismo animal, hipnosis, ciencia y literatura, medicina y literatura.

KEY WORDS: mesmerism, magnetism, hypnosis, science and literature, medicine and literature. 


\section{Introducción}

La imagen pública de la ciencia juega un papel fundamental en el propio desarrollo de las disciplinas científicas. De hecho, la interpretación social y popular de una teoría científica influye en la evolución científica y en los cambios sociales que acontecen en una comunidad científica determinada ${ }^{1}$. En este sentido, el estudio del papel que juegan los mediadores en los procesos de circulación y comunicación del conocimiento científico resulta especialmente útil e interesante para entender el modo en que el público demanda y accede a este tipo de conocimiento. Unos procesos de circulación del conocimiento en los que la literatura de ficción adquirió a lo largo de los siglos XIX y XX una importancia capital ${ }^{2}$.

En el presente trabajo nos acercamos a la imagen social o pública del fenómeno conocido popularmente primero como mesmerismo o magnetismo animal y después como hipnosis, entre otros tantos nombres según diferentes autores $^{3}$. El término «hipnosis» surge de las ideas de Braid, que llamó «hipnotismo» a la forma extrema que presentaba amnesia tras el proceso del «sueño nervioso» ${ }^{4}$. Los términos más usados y difundidos en la literatura tanto académica como de ficción son mesmerismo, magnetismo animal e hipnosis y los libros de la historia de estas materias suelen diferenciar dos grandes bloques temáticos (a modo de etapas): mesmerismo/magnetismo animal por un lado e hipnosis por otro ${ }^{5}$.

Parece fuera de toda duda que la ficción literaria refleja una parte importante de la cultura científica de una época. Como apuntó Baudelaire:

«La unidad animal, la unidad de un fluido universal, la unidad de la materia; todas estas recientes teorías, por una extraña coincidencia, han

1. Pyenson, Lewis; Sheets-Pyenson, Susan. Servants of nature: A history of scientific institutions, enterprises, and sensibilities. New York: W. W. Norton \& Company; 1999.

2. Nieto-Galán, Agusti. Los públicos de la ciencia: expertos y profanos a través de la historia. Madrid: Marcial Pons; 2011.

3. En 1883, la Enciclopedia Británica diferencia entre magnetismo animal, mesmerismo e hipnosis aunque todo bajo el término de magnetismo. Spencer, Thomas, ed. The Encyclopedia Britannica: A dictionary of arts, sciences, and general literature. Ninth edition. Volume XV. New York: Charles Scribner's Sons; 1883.

4. López Piñero, José María. Del hipnotismo a Freud. Orígenes históricos de la psicoterapia. Madrid: Alianza Editorial; 2002.

5. Así lo encontramos en Gauld, Alan. A history of hypnotism. New York: Cambridge University Press; 1992 y en Montiel, Luis; González de Pablo, Ángel, coords. En ningún lugar, en parte alguna. Estudios sobre la historia del magnetismo animal y del hipnotismo. Madrid: Frenia; 2003. 
entrado, de alguna manera, en la mente de los poetas y de los científicos al mismo tiempo» ${ }^{6}$.

En este sentido, durante el siglo XIX y primeras décadas del XX, el número de autores que usaron el mesmerismo y la hipnosis como recurso, central o periférico, para sus historias de ficción es bastante amplio, entre otros: E.T.A Hoffmann, Gustave Flaubert, Honore de Balzac, Guy de Maupassant $^{7}$, Charles Dickens, Wilkie Collins, Nathaniel Hawthorne y Mark Twain ${ }^{8}$. Para este trabajo hemos escogido tres obras literarias donde el magnetismo animal desempeña un papel central. The Facts in the Case of Mr. Valdemar (1845) ${ }^{9}$ de Edgar Allan Poe (1809-1849), The Great Keinplatz Experiment $(1885)^{10}$ de Arthur Conan Doyle (1859-1930) y Trilby (1894) ${ }^{11}$ de George Du Maurier (1834-1896). Como más adelante veremos, en los casos de Poe, Doyle y Du Maurier podemos hablar de magnetismo animal o mesmerismo mientras que no sería adecuado hablar de hipnosis ${ }^{12}$.

En el presente trabajo intentaremos mostrar la imagen del mesmerismo que nos proporcionan las tres obras analizadas. Mostraremos cuál es el estereotipo del magnetizador y los usos que observamos del mesmerismo. Nos acercaremos a los espacios y actores del trasunto mesmerico presentado

6. La cita aparece en el prefacio a la traducción al francés, hecha por Baudelaire, de Mesmeric Revelation (1844) de Poe; la traducción al español de la cita la he tomado de Pis-Díez, Gustavo. Hipnotismo y literatura: el caso de E. A. Poe. In: Fuentenebro, Filiberto, ed. Historia de la psiquiatría en Europa. Madrid: Frenia; 2003, p. 421-434 (425).

7. Ellenberger, Henri. El descubrimiento del inconsciente. Historia y evolución de la psiquiatría dinámica. Madrid: Gredos; 1976.

8. Hartman, Donald. Hypnotic and mesmeric themes and motifs in selected English-language novels, shorts stories, plays and poems, 1820-1983. Bulletin of Bibliography. 1987; 44: 156-166.

9. Poe, Edgar Allan. The facts in the case of Mr. Valdemar. The American Review: A Whig Journal. 1845; 2: 561- 565. He usado esta publicación.

10. Doyle, Arthur Conan. The great Keinplatz experiment. Belgravia Magazine. Julio 1885. Más tarde el relato aparece en una obra de 3 volúmenes titulada Dreamland and Ghostland: An original collection of tales and warnings from the borderland of substance and shadow (1888). He usado las publicaciones: Doyle, Arthur Conan. Historias del crepúsculo y de lo desconocido. Madrid: Valdemar; 1998, y Doyle, Arthur Conan. The great Keinplatz experiment. In: The Captain of the Pole Star and other stories. New Zealand: Floating Press; 2010, p. 88-113.

11. Du Maurier, George. Trilby. Harper's Magazine. Enero 1894. He usado las publicaciones: Du Maurier, George. Trilby. Barcelona: Funambulista; 2008 y Du Maurier, George. Trilby. London: Penguin; 1995.

12. En la traducción al castellano del relato de Doyle citada anteriormente en Doyle, n. 10, p. 49-71, se usan indiscriminadamente las expresiones mesmerismo e hipnosis; no es así en la versión en inglés también citada en Doyle, n. 10, p. 88-113, en la cual solo encontraremos expresiones relativas al mesmerismo. 
en los relatos. Por último, tendremos en cuenta la recepción por parte del público de estas historias y las relaciones con los conocimientos mesmericos que tenían los autores de éstas.

Ya en el año 1892, Karl Emil Franzos editó un libro titulado Suggestion and Literary Fiction donde, mediante opiniones de expertos como Otto Binswanger, Friedrich Fuchs y Krafft-Ebing, intentó prevenir a la población del abuso y distorsiones del tema hipnótico en la literatura de ficción ${ }^{13}$. En 1901, Joseph Jastrow, profesor de psicología en la Universidad de WisconsinMadison, publicó un libro titulado Fact and Fable in Psychology, donde articulaba una historia del magnetismo animal y de la hipnosis poniendo énfasis en los mitos y creencias erróneas de la población en estas materias ${ }^{14}$.

En la actualidad, investigadores académicos, dentro de la disciplina de la psicología, publican artículos y libros sobre los mitos populares de la hipnosis intentando poner de manifiesto las imágenes distorsionadas, y ampliamente difundidas, referentes a este fenómeno ${ }^{15}$. Las implicaciones de este tipo de trabajos cobran importancia por el hecho, confirmado por la investigación, de que las actitudes y creencias sobre la hipnosis juegan un papel esencial en la eficacia del tratamiento con técnicas hipnóticas ${ }^{16}$. Bastantes autores coinciden en afirmar que, actualmente, la población en general posee una visión equivocada y sesgada hacia lo negativo de la hipnosis ${ }^{17}$. Esta imagen distorsionada del proceso hipnótico, y del hipnotizador, proviene de los espectáculos circenses de hipnosis (stage hypnosis), del cine y de la televisión ${ }^{18}$. Es obvio que, siguiendo el rastro del cine como influencia fundamental en las creencias y actitudes sobre la hipnosis por parte del público, llegaremos a la literatura de ficción, origen de los argumentos y guiones para la mayoría de las películas del género. Sirva de ejemplo el relato

13. Andriopoulos, Stefan. Possessed: Hypnotic crimes, corporate fiction, and the invention of cinema. Chicago: The University of Chicago Press; 2008.

14. Jastrow, Joseph. Hypnotism and its antecedents. In: Jastrow, Joseph. Fact and fable in psychology. London: Macmillan; 1901, p. 171-231.

15. Un artículo de revisión de este tipo de literatura es Barling, Norman; De Lucchi, Daniele. Knowledge, attitudes, and beliefs about clinical hypnosis. Australian Journal of Clinical and Experimental Hypnosis. 2004; 32 (1): 36-52.

16. Hawkins, Russell; Bartsch, Joanna. The effects of an educational lecture about hypnosis. Australian Journal of Clinical and Experimental Hypnosis. 2000; 28 (1): 82-99.

17. Large, Robert; James, Frances. Public expectations of hypnosis. Australian Journal of Clinical and Experimental Hypnosis. 1991; 19 (1): 103-106.

18. Johnson, Myles; Hauck, Coleen. Beliefs and opinions about hypnosis held by the general public: A systematic evaluation. American Journal of Clinical Hypnosis. 1999; 42 (1): 10-18. 
aquí escogido de Poe, La verdad sobre el caso del señor Valdemar ${ }^{19}$, que ha sido objeto de numerosas versiones cinematográficas, o la más reciente The Manchurian Candidate (2004) del director Jonathan Demme, basada en la novela homónima de 1959 de Richard Condon ${ }^{20}$.

Entre los grandes mitos de la hipnosis encontramos: la creencia de que cualquier persona es altamente hipnotizable, el miedo a perder totalmente el control y realizar actos inmorales o delictivos en estado de hipnosis, la imposibilidad de recordar aquello que ocurrió en estado hipnótico, el peligro de volverse loco o demente en estado de hipnosis y la posibilidad de experimentar fenómenos paranormales como la clarividencia, viajes astrales, telepatía y regresión a vidas pasadas ${ }^{21}$. Sin embargo, en la otra cara de la moneda, nos encontramos que:

«todo aquello que, desde hace dos siglos, ha podido salvarse y transmitirse de la herencia del magnetismo; lo que finalmente ha podido conseguir una especie de victoria "de fondo" en el terreno de las sensibilidades culturales y morales (pero evidentemente no en los saberes oficiales) ha sido transmitido por la literatura, la poesía, el teatro, las artes, la obra de algunos filósofos y todo el movimiento ordinario de las representaciones banales» ${ }^{22}$.

Desde la anterior perspectiva, el estudio de las obras literarias de ficción se convierte en fuente inestimable para el historiador; aún más para el historiador de materias que han sido olvidadas o borradas por la triunfante cultura cientificista y para el cual, parafraseando a Walter Benjamin, la literatura se convierte en un sistema de historia.

\section{La verdad sobre el caso del señor Valdemar, Edgar Allan Poe}

El magnetismo animal fue introducido en Estados Unidos en 1836 por el francés Charles Poyen, que impartió una serie de conferencias sobre el

19. Carrillo, Juan Luis. ¡Detened la muerte! Un arquetipo amenazante como recurso literario y cinematográfico. In: Montiel; González de Pablo, n. 5, p. 301-324.

20. Tudor, Andrew. Monsters and mad scientists: A cultural history of the horror movie. Oxford: Basil Blackwell; 1989.

21. Capafons, Antonio. Hipnosis clínica: una visión cognitivo-comportamental. Papeles del Psicólogo. 1998; 69: 71-88.

22. Peter, Jean-Pierre. Lo que los magnetizadores nos han enseñado (de Mesmer a Puysegur). In: Montiel; González de Pablo, n. 5, p. 35-61 (50). 
tema inicialmente en Boston y más tarde en diferentes lugares como Rodhe Island, Nueva Inglaterra y Nueva York ${ }^{23}$. A partir de aquí, el mesmerismo se propagó rápidamente por todo Estados Unidos de forma exitosa. Este éxito se explica por el hecho de que el país no poseía unos poderes institucionales conservadores (como sí había ocurrido en Francia) que pudieran articular una reacción crítica al magnetismo animal ${ }^{24}$. La aceptación del mesmerismo también se benefició de la mentalidad religiosa de la población que creía en la existencia de un espíritu contenido en el cuerpo carnal humano y en la conexión de éste con influencias invisibles o inmateriales ${ }^{25}$. El magnetismo animal tuvo fuertes afinidades con otros movimientos (como el vegetarianismo, el amor libre, la homeopatía y la frenología) que se estaban desarrollando en Estados Unidos en esos momentos, lo que sin duda contribuyó a su difusión y aceptación pública ${ }^{26}$.

Así las cosas, las conferencias sobre magnetismo animal, las demostraciones públicas del proceso mesmerico, los tratamientos basados en estas ideas y las publicaciones sobre el tema se propagaron con celeridad por todo Estados Unidos. Las publicaciones sobre mesmerismo de autores americanos comenzaron muy pronto y ya en 1860 había más de 50 títulos sobre el tema entre libros, revistas y manuales ${ }^{27}$.

El movimiento mesmerico en Estados Unidos se fue apagando poco a poco para desaparecer a finales del siglo XIX. No hubo oposición institucional o académica, como en Francia, pero la sociedad norteamericana, tan fascinada por el mesmerismo en un principio, se acabó cansando de éste. Los valores masculinos ${ }^{28}$, materialistas y utilitaristas de la población ahogaron al mesmerismo que terminó diluido, filtrado o reciclado en nuevos movimientos como el espiritismo, o en diferentes escuelas híbridas como

23. Carlson, Eric. Charles Poyen brings mesmerism to America. Journal of the History of Medicine and Allied Sciences. 1960; 25 (2): 121-132.

24. Mills, Bruce. Poe, Fuller, and the mesmeric arts: Transition states in the American Renaissance. Columbia: University of Missouri Press; 2006, este autor señala que desde 1936 a 1941 sólo encontramos 5 artículos críticos con el magnetismo animal, todos ellos escritos por médicos y aparecidos en la revista Boston Medical and Surgical Journal.

25. Fuller, Robert. Mesmerism and the American cure of souls. Philadelphia: The University of Pennsylvania Press; 1982.

26. Gauld, n. 5, p. 180.

27. Schmit, David. Re-visioning antebellum American psychology: The dissemination of mesmerism, 1836-1854. History of Psychology. 2005; 8 (4): 403-434.

28. El mesmerismo fue criticado por «feminizar» a la población masculina. 
la fundada por Phineas Quimby o la Christian Science fundada por una paciente de Quimby conocida como Mary Baker Eddy ${ }^{29}$.

En 1837, en Filadelfia, se publicó un libro anónimo titulado The Philosophy of Animal Magnetism by a Gentleman of Philadelphia, dedicado a «especially the Ladies of the United States of America» ${ }^{30}$. Casi cien años más tarde, en 1928, la autoría del libro fue atribuida a Edgar Allan Poe por el historiador Joseph Jackson ${ }^{31}$. Obviando los datos que hicieron que Jackson atribuyera la autoría a Poe, propios de una investigación histórica con más o menos rigor ${ }^{32}$, la idea de Poe como el autor de un tratado de magnetismo animal no resulta del todo quimérica, considerando que entre 1844 y 1845 , escribió tres relatos cortos donde el mesmerismo juega un papel central. Se trata del relato de Valdemar, ya citado anteriormente, $A$ Tale of the Ragged Mountains (1844) ${ }^{33}$ y Mesmeric Revelation (1844) ${ }^{34}$. Además Poe reseñó los libros Human Magnetism: Its Claims to Dispassionate Inquiry (1845) de W. Newnham y Facts in Mesmerism (1840) de C. H. Townshend ${ }^{35}$. Poe comparó ambos libros, elogiando a Townshend y siendo más crítico hacia Newnham (que se había distanciado de las ideas del primero) ${ }^{36}$. No

\footnotetext{
29. Schmit, n. 27, p. $417-422$.

30. The philosophy of animal magnetism by a gentleman of Philadelphia. Philadelphia: Merrihew\&Gunn; 1837, p. 3.

31. Aunque en la actualidad podemos encontrar diferentes ediciones del libro en las que Poe aparece como autor la autoría del libro continúa siendo controvertida y discutida por diferentes autores.

32. Las razones de Jackson para atribuir la autoría del libro a Poe se explican en la introducción del libro a partir de la versión de 1928: Poe, Edgar Allan; Jackson, Joseph. The philosophy of animal magnetism by a gentleman of Philadelphia. Whitefish: Kessinger Publishing; 2010, p. 5-14.

33. Poe, Edgar Allan. A tale of the Ragged mountains. Godey's Lady's Book. 1844; 28: 177-181.

34. Poe, Edgar Allan. Mesmeric revelation. Columbian Lady's and Gentleman's Magazine. 1844; 2: 67-70. Dos trabajos donde se trata extensamente este relato de Poe son Pis-Díez, n. 6, p. 428-433 (junto al relato de Valdemar) y Van Schlun, Betsy. Edgar Allan Poe's universe of suggestion. In: Science and the imagination: Mesmerism, media and the mind in nineteenthcentury English and American literature. Berlin: Galda Wilch Verlag; 2007, p. 253-259.

35. Lind, Sidney E. Poe and mesmerism. PMLA. 1947; 62 (4): 1077-1094.

36. El desencuentro teórico entre Newnham y Townshend se produjo en relación al origen y la substancialidad del magnetismo animal. Para Townshend el magnetismo animal era algo parecido a luz/calor/electricidad y existía independientemente del magnetizador y del magnetizado. La explicación de Newnham era más psicológica y daba más importancia a la figura del magnetizador.
} 
obstante, consideró la obra de Newnham como de gran importancia y mucho mérito ${ }^{37}$.

El relato de Valdemar fue recibido por la sociedad como un caso verídico cuando fue reeditado bajo el título The Last Conversation of a Somnambule (1845) en la respetable revista científica London Popular Record of Modern Science $^{38}$. Este hecho no es sorprendente si tenemos en cuenta la práctica común de algunos médicos, sobre todo en el período romántico alemán ${ }^{39}$, de publicar sus casos clínicos escritos en un estilo y con unas características narrativas similares a las usadas por Poe en su relato ${ }^{40}$. La consideración de Poe como un entendido en mesmerismo, llevó a autores como Andrew Jackson Davis (1826-1910) a citarlo profusamente en sus obras sobre magnetismo animal como por ejemplo en The Great Harmonia $(1852)^{41}$.

En La verdad sobre el caso del señor Valdemar, Poe nos presenta un experimento mesmerico consistente en la práctica del magnetismo animal en un voluntario, el señor Valdemar, en el umbral de su muerte por tuberculosis. El experimento es realizado por un médico amigo de Valdemar. Mientras Valdemar cae en trance, afirma primero que está muriendo, luego que está muerto, quedando en un estado mesmerico durante siete meses, en los cuales carece de pulso y respiración perceptible, y su piel se aprecia pálida y fría. Finalmente, Valdemar es despertado y su cuerpo se descompone inmediatamente ${ }^{42}$. Poe usa durante todo el relato palabras y tono científico demostrando las preocupaciones científicas que se desarrollaron durante la segunda mitad del siglo XIX (realismo, cientificismo, positivismo). En este sentido es memorable la descripción que realiza del avanzado estado de la enfermedad del señor Valdemar ${ }^{43}$. Así las cosas, tenemos un experimento con un objetivo científico «to what extend, or for how long a period, the

\footnotetext{
37. Mills, n. 24, p. 56-58.

38. Enns, Anthony. Mesmerism and the electric age: From Poe to Edison. In: Willis, Martin; Wynne, Catherine, eds.Victorian literary mesmerism. Amsterdam: Rodopi B.V.; 2006, p. 61-82.

39. Esta práctica no se limitó a este periodo. Consúltese, por ejemplo: Puysegur, Jacques de Chastenet; Peter, Jean-Pierre, ed. Un somnambule désordonné? Journal du traitement magnétique du jeune Hébert. Le Plessis-Robinson: Institut Synthelabo; 1999.

40. Montiel; González de Pablo, n. 5, p. 101-138.

41. Damon, Foster. Thomas Holly Chivers friend of Poe. New York: Russell\&Russell; 1930.

42. Poe, n. 9, p. 561- 565.

43. Como algunos críticos han señalado, entre ellos Lind, n. 35, p. 1093, los detalles expuestos por Poe relativos a los órganos internos del señor Valdemar no podían saberse, con los medios médicos de la época, sino tras una autopsia.
} 
encroachments of Death might be arrested by the process» ${ }^{44}$. El experimento es dirigido por un médico y su ayudante, que es estudiante de medicina. También colaboran en el experimento los médicos que estaban tratando al señor Valdemar de su enfermedad. El mesmerizador se nos presenta como un hombre de ciencia, racional y con buenas intenciones (el avance de la ciencia) que no duda en poner fin al experimento cuando el propio Valdemar se lo pide. El mesmerizador no se nos presenta como un ser con oscuras intenciones, ni como una persona malvada ni amoral, los peligros no vienen de él sino, en todo caso, de unos conocimientos científicos puestos en práctica de forma experimental. Del sujeto mesmerizado se nos dice que «His temperament was markedly nervous, and rendered him a good subject for mesmeric experiment» ${ }^{45}$. El método del mesmerizador consiste en «exchanging, however, the lateral passes for downward ones, and directing my gaze entirely into the right eye of the sufferer» ${ }^{46} \mathrm{y}$ las señas del estado mesmerico en Valdemar son al principio una completa rigidez de los miembros para pasar a un «unusually perfect state of mesmeric trance» ${ }^{4}$.

La reseña favorable que Poe realizó del libro Facts in Mesmerism (1840) de Townshend apunta a que este texto fue una de las influencias mesmericas de Poe. La influencia queda patente en el hecho de que Poe usó en el relato de Valdemar hasta cinco veces la palabra sleepwaker. En su libro, Townshend señalaba más apropiado usar la denominación sleepwaker en lugar de somnambulism o sleepwalker para designar tales fenómenos. Una idea que no reflejó ningún otro autor ni texto sobre mesmerismo de la época, lo que avala que ésta fue la fuente de Poe ${ }^{48}$. En el mismo sentido abunda el hecho de que Townshend publicó un caso en la revista Notice en 1844 en el que relató cómo consiguió prolongar dos meses la vida de un moribundo mediante mesmerismo ${ }^{49}$.

El influyente trabajo de James Braid Neurypnology, or the Rationale of Nervous Sleep considered in relation with animal magnetism apareció en 1843, dos años antes de la publicación del relato de Valdemar. Sin embargo, no hemos encontrado indicios explícitos ni implícitos de que Poe tuviera en

\footnotetext{
44. Poe, n. 9, p. 561.

45. Poe, n. 9, p. 561.

46. Poe, n. 9, p. 562.

47. Poe, n. 9, p. 563.

48. Lind, n. 35, p. 1089.

49. Lind, n. 35, p. 1091.
} 
cuenta esta obra para la creación de sus relatos, ya que en ningún momento Poe usa las expresiones «hipnosis» $\mathrm{O}$ «hipnotismo» ${ }^{50}$. Debemos anotar que a finales del siglo XIX existía cierta flexibilidad en el uso de un término $\mathrm{u}$ otro, sobre todo por autores para los cuales el magnetismo animal era lo mismo que la hipnosis. Sirvan de ejemplo dos textos con títulos bien explícitos: How to Hypnotise: Including the Whole Art of Mesmerism (1890) de John Barter y Human Magnetism or How to Hypnotise: A Practical Handbook for Students of Mesmerism (1897) de James Coates. En este sentido, la traducción al español del relato de Valdemar realizada por Julio Cortázar puede confundirnos ya que tradujo sistemáticamente mesmerism por hipnosis ${ }^{51}$, no así la versión de Julio Gómez de la Serna que tradujo mesmerism por mesmerismo y/o magnetismo animal ${ }^{52}$.

Sarah Whitman, amiga de Poe, reflejó en una de sus cartas el profundo background mesmerico que Poe usó en sus relatos: «People seem to think there is something uncanny about Poe, and the strangest stories are told about his mesmeric experiences, at the mention of which he always smiles» ${ }^{53}$. Estos relatos son vistos por la crítica literaria como precursores de la ciencia ficción por descansar el nervio de la historia en el uso de conocimientos científicos de una época (el magnetismo animal), y fueron recibidos, en el momento de su aparición, como legítimas contribuciones al campo de la ciencia. Algunos autores, como Bruce Mills, señalan que los conocimientos mesmericos de Poe, tomados como una filosofía que le cambió profundamente su postura ante la vida, fueron los que precipitaron el nacimiento del relato corto en literatura $^{54}$. Sin embargo, las intenciones de Poe al escribir estos relatos permanecen ambiguas. Ante las alabanzas a Mesmeric Revelation (1844) por parte del reverendo George Bush, importante líder del movimiento swedenborgiano, Poe declaró que «The story is a pure fiction from beginning to $e n d{ }^{5} 5$.

\footnotetext{
50. Falk, Doris V. Poe and the power of animal magnetism. PMLA. 1969; 84 (3): 536-546.

51. Poe, Edgar Allan. La verdad sobre el caso del señor Valdemar. In: Cuentos de imaginación y misterio. Barcelona: Libros de Zorro Rojo; 2009, p. 85-96.

52. Poe, Edgar Allan. El caso del señor Valdemar. In: Obras selectas de Edgar Allan Poe. Barcelona: Orbis; 1983, p. 117-126.

53. Poe, Edgar Allan; Mabbott, Thomas, ed. Collected works of Edgar Allan Poe. Cambridge: The Belknap Press of Harvard University Press; 1978, vol. 3, p. 1028.

54. Mills, n. 24, p. 43-66.

55. Poe, n. 53, p. 1367.
} 


\section{El gran experimento de Keinplatz, Conan Doyle}

El gran experimento de Keinplatz (1855) de Doyle se sitúa en tierras germanas, concretamente en una región al sur, en la universidad de Keinplatz. En los primeros años de la década de 1790, el magnetismo animal había llegado a ser en Alemania una materia respetable y recibía un tratamiento académico junto con las pre-románticas teorías sobre la luz y la electricidad ${ }^{56}$. Otros, como Eberhard Gmelin en su obra Neue Untersuchungen bei den thierischen Magnetismus (1789), explicaron el magnetismo animal basándose en la fisiología. No obstante, fue durante el romanticismo alemán cuando el magnetismo se desarrolló hasta alcanzar sus más interesantes expresiones ${ }^{57}$. Los autores del romanticismo alemán se sintieron fascinados por el magnetismo animal y lo usaron como tema central de sus creaciones literarias, siendo ellos los que acuñaron y popularizaron el término «mesmerismo» ${ }^{58}$. Una de las razones del éxito del magnetismo animal en Alemania fue la correspondencia filosófica, metafísica y antropológica con el espíritu de la Naturphilosophie, articulada por Wilhelm Schelling en su obra Primer proyecto de la filosofía de la naturaleza $(1797)^{59}$. El magnetismo animal durante el siglo XIX se desarrolló en Alemania gracias a autores como Dietrich Georg Kieser, Carl August Eschenmayer ${ }^{60}$, Carl Alexander Ferdinand Kluge, Friedrich Hufeland o Johann Carl Passavant, por citar algunos de los más importantes ${ }^{61}$. Alan Gauld, en su texto dedicado al magnetismo animal en Alemania introduce a Justinus Kerner ${ }^{62}$. Su texto Die Seherin von Prevorst (1829), donde narró sus experiencias con la vidente de Prevorst (Friederike Hauffe), se convirtió en obra de referencia de los círculos espiritistas ${ }^{63}$. De esta manera, Gauld

\footnotetext{
56. Gauld, n. 5, p. 81-83.

57. Beguin, Albert. El alma romántica y el sueño. México: Fondo de Cultura Económica; 1954.

58. Barkhoff, Jürgen. Romantic science and psychology. In: Saul, Nicholas, ed. The Cambridge companion to German Romanticism. New York: Cambridge University Press; 2009, p. 209-225.

59. Montiel, Luis. Daemoniaca. Curación mágica, posesión y profecía en el marco del magnetismo animal romántico. Barcelona: mra; 2006.

60. Kieser y Eschenmayer fueron co-directores de la revista Archiv für den thierischen Magnetismus (1817-1824).

61. He seguido en este repaso (brevísimo y general) de autores a Gauld, n. 5, p. 141-144. Para una aproximación más detallada del tema puede verse Montiel, Luis. Magnetizadores y sonámbulas en la Alemania romántica. Madrid: Frenia; 2008.

62. Gauld, n. 5, p. 141-162.

63. Montiel, Luis. Síntomas de una época: magnetismo, histeria y espiritismo en la Alemania romántica. Asclepio. 2006; 58 (2): 11-38.
} 
(a través de Kerner) establece la conexión entre el magnetismo animal y el espiritismo. En El gran experimento de Keinplatz, Doyle también establece esta conexión del magnetismo animal con el espiritismo, aún más, conecta al primero con la escuela swendenborgiana y con los rosacruces ${ }^{64}$.

Arthur Conan Doyle tuvo una vida bastante peculiar. De origen humilde, estudió medicina y durante muchos años vivió frugalmente, se hizo a la mar en un barco pesquero, estuvo en varias guerras, vivió largas temporadas en el extranjero, tuvo una breve carrera política, estudió (durante más de treinta años) los fenómenos espiritistas y se convirtió a esta doctrina, escribió bastantes novelas históricas al estilo de las grandes narrativas (lo que él consideraba su mejor obra). Sin embargo, será siempre recordado, hecho que a él le disgustaba, por ser el creador de Sherlock Holmes ${ }^{65}$.

Doyle comenzó a interesarse por el espiritismo en la década de 1880. Pasó noches en casas encantadas, asistió a numerosas sesiones de médiums, formó parte de la Society for Psychical Research de Londres ${ }^{66}$, tuvo contactos con extraños personajes pertenecientes a los rosacruces, a la escuela de Swedenborg y a grupos mucho más secretos ${ }^{67}$. En el frontispicio de su Historia del espiritismo (1926), debajo del nombre del autor aparecen los siguientes títulos: Presidente del Colegio Británico de Ciencia Psíquica, Presidente Honorario de la Federación Espiritista Internacional y Presidente de la Alianza Espiritista de Londres ${ }^{68}$.

Todo este background espiritista se reflejó en algunos relatos de Sherlock Holmes, en particular en The Man with the Twisted Lip (1891) y The Sign of Four (1890) en los que se describen sesiones espiritistas ${ }^{69}$. Doyle escribió tres relatos donde el mesmerismo tenía un papel central: The great Keinplatz experiment (1885), John Barrington Cowles (1884) y The Parasite (1894). El argumento de John Barrington Cowles y The Parasite es muy similar: una mujer (el estereotipo de femme fatale) mesmeriza a un joven para conseguir

\footnotetext{
64. Doyle, n. 10, p. 56.

65. Doyle, Arthur Conan. Memorias y aventuras. Madrid: Valdemar; 1924.

66. Haynes, Renee. The Society for Psychical Research 1882-1982: A history. London: MacDonald; 1982.

67. Stavert, Geoffrey. A study in Southsea: The unrevealed life of doctor Arthur Conan Doyle. Portsmouth: Milestone Publications; 1987.

68. Doyle, Arthur Conan. Historia del espiritismo. Madrid: Eyras; 1926.

69. Grimes, Hilary. Sensitive to the invisible: Photography and the supernatural in the Holmes stories, Arthur Conan Doyle's spiritualism, and Francis Galton's composite portraits. In: The late Victorian Gothic: Mental science, the uncanny, and scenes of writing. Cornwall: Ashgate; 2011, p. 37-60.
} 
su amor y ambos relatos terminan trágicamente ${ }^{70}$. The Parasite y Trilby de Du Maurier se publicaron en 1894. El éxito de Trilby eclipsó la novela de Doyle, que pasó desapercibida. Más tarde, Doyle renegó de ella e intentó que las editoriales y biógrafos la excluyeran de su curriculum bibliográfico ${ }^{71}$. Sin embargo, los tres relatos de Doyle sobre mesmerismo tienen el mérito innegable de contraponerse a algunas de las convenciones argumentales más estereotipadas de la literatura de ficción mesmerica (el relato de Keinplatz no es de terror sino cómico y en John Barrington Cowles y The Parasite el villano magnetizador es una mujer y la víctima inocente es un hombre).

En el relato de Doyle, el profesor Baumgarten de la universidad de Keinplatz está interesado en «to build a new exact science which should embrace mesmerism, spiritualism, and all cognate subjects» ${ }^{72}$. En este sentido, Baumgarten y su alumno Hartmann preparan un experimento mesmerico, para probar que el alma puede existir sin el cuerpo y viajar fuera de este, que consiste en:

«to mesmerise one of my pupils. I shall then mesmerise myself in a manner which has become easy to me. After that, if my theory holds good, my spirit will have no difficulty in meeting and communicating with the spirit of my pupil, both being separated from the body» ${ }^{73}$.

Hartmann había sido magnetizado por el profesor Baumgarten con anterioridad (en al menos cuatro ocasiones) y en estado cataléptico había sido sometido a descargas eléctricas. En Inglaterra, este tipo de experimentos con personas mesmerizadas fueron habituales en la época, incluyendo las descargas eléctricas, pinchazos con alfileres o vertidos de ácido en la piel del sujeto ${ }^{74}$. En cuanto al experimento principal del relato de Doyle, encontramos un cierto parecido con los experimentos del médico Herbert Mayo, profesor de anatomía en el King College de Londres, que durante 1838 realizó experimentos con el objetivo de probar la existencia del alma y su independencia de la vida orgánica ${ }^{75}$.

70. Van Schlun, Betsy. Arthur Conan Doyle: A case of identity. In: Van Schlun, n. 34, p. 251-253.

71. Booth, Martin. The doctor, the detective and Arthur Conan Doyle: A biography of Arthur Conan Doyle. London: Thomas Dunne Books; 1997.

72. Doyle, n. 10, p. 89.

73. Doyle, n. 10, p. 92.

74. Winter, Alison. Mesmerized: Powers of mind in Victorian Britain. Chicago: University of Chicago Press; 1998.

75. Podmore, Frank. Mesmerism and Christian Science: A short history of mental healing. Cambridge: Cambridge University Press; 1909. 
El experimento del profesor Baumgarten se lleva a cabo en el laboratorio de fisiología de la Universidad de Keinplatz, está previsto que los resultados se publiquen en una revista médica y acuden a presenciarlo todos los grandes hombres de ciencia del sur de Alemania. También acuden al experimento un profesor de Londres experto en los centros cerebrales, un ministro swedenborgiano en busca de pruebas para elucidar la doctrina Rosacruz y numerosos adeptos del espiritismo.

El método magnético de Baumgarten es bastante sencillo: «With a few rapid passes the Professor mesmerised the young man» ${ }^{76}$. Para magnetizarse a sí mismo el profesor usa una bola de cristal en la que «concentrating his gaze upon it and making a strong mental effort, he succeeded in throwing himself into the same condition ${ }^{77}$. Al regresar del trance mesmerico, se genera una confusión en la sala del experimento, el profesor parece aturdido y es su alumno quien salva la situación con el público, aun así, el experimento es visto como un fracaso ya que no se han podido demostrar los objetivos iniciales al público. En realidad, lo que ha ocurrido es que el espíritu del profesor ha tomado como suyo el cuerpo del alumno y el espíritu del alumno ha hecho lo mismo con el cuerpo del profesor. Así las cosas, el cuerpo del profesor llevado por el espíritu del alumno se va de juerga con sus amigos y el cuerpo del alumno llevado por el espíritu del profesor regresa a su casa, generándose diversas situaciones equívocas y cómicas. Al final, ambos se dan cuenta de lo ocurrido y mediante un nuevo trance mesmerico sus espíritus regresan a sus cuerpos originales.

Es habitual encontrar autores de literatura de ficción, sobre todo a finales del siglo XIX, que en sus novelas establecieron conexiones entre el mesmerismo y el espiritismo o con otras de las tradiciones llamadas ocultas ${ }^{78}$. No sólo los autores de ficción han realizado dichas conexiones. En 1847, el reverendo George Bush publicó Mesmer and Swedenborg: The Relation of the Developments of Mesmerism to the Doctrines and Disclosures of Swedenborg. Ellenberger ve en la figura del chamán y en su poder con los espíritus un antepasado remoto del magnetizador ${ }^{79}$ y Crabtree considera que Mesmer era

\footnotetext{
76. Doyle, n. 10, p. 97.

77. Doyle, n. 10, p. 97.

78. Andriopoulos, n. 13, p. 39.

79. Ellenberger, n. 7, p. 20-73.
} 
un estudioso de las enseñanzas de los Rosacruces ${ }^{80}$. Doyle manejaba todas esas fuentes ocultas (Rosacruces, Swedenborg y espiritismo) con soltura. Sin embargo, su principal fuente en relación a los temas mesmericos fue el libro Animal Magnetism (1887) de Alfred Binet y Charles Fere ${ }^{81}$.

En un ensayo inédito, Doyle mostró su particular y elaborada forma de entender el magnetismo animal:

«A man is impelled to do some act by the irresistible action of a mesmeric suggestion which may have been made some months before. He would scout the idea that the impulse came from without, and yet we know that it is so. How can we tell that all our actions are not of this nature? What appears to us to be our own choice may prove really to have been as unalterable and inexorable as fate, the unavoidable result of the sum total of suggestions which are acting upon us» ${ }^{82}$.

\section{Trilby, Du Maurier}

Aunque Du Maurier nació en Francia pasó largas temporadas en Inglaterra, y su producción artística (como ilustrador) y literaria comenzó y se desarrolló en Londres ${ }^{83}$. Los historiadores del magnetismo animal han documentado la recepción de éste en Inglaterra a partir de $1785^{84}$. No obstante, se considera que fue en 1837, cuando el magnetizador Jules Du Potet exportó el magnetismo animal a tierras británicas. Su éxito fue limitado ya que no hablaba inglés, por lo que la campaña se limitó a demostraciones públicas ${ }^{85}$. En 1840, otro renombrado magnetizador francés Charles Lafontaine continuó la misión de Du Potet (que había permanecido casi dos años en Londres) de expandir el magnetismo en Inglaterra, impartiendo conferencias además de

80. Crabtree, Adam. From Mesmer to Freud: Magnetic sleep and the roots of psychological healing. New Haven: Yale University Press; 1993.

81. Nordon, Pierre. Conan Doyle: A biography. London: Holt; 1966.

82. Cita tomada de Nordon, n. 81, p. 151.

83. Para una historia del magnetismo animal en Francia puede seguirse a: Gauld, n. 5; Peter, n. 22, p. 35-61 (de Mesmer a Puysegur) y Peter, n. 39, p. 9-88 (sobre Puysegur). Sobre el magnetismo más tardío puede verse: Darnton, Robert. Mesmerism and the end of the Enlightenment in France. Cambridge: Harvard University Press; 1968 y Monroe, John. Laboratories of faith: Mesmerism, spiritism, and occultism in Modern France. Ithaca: Cornell University Press; 2008.

84. Gauld, n. 5, p. 197. Fara, Patricia. Sympathetic attractions: Magnetic practices, beliefs, and symbolism in eigteenth-century England. Princeton: Princeton University Press; 1996.

85. Winter, n. 74, p. 40-45. 
realizar demostraciones públicas ${ }^{86}$. La sociedad inglesa pronto se fascinó por estas nuevas ideas, aunque la élite médica y académica demostró importantes recelos dada su procedencia francesa ${ }^{87}$. John Elliotson, un joven y afamado médico y profesor del University College de Londres, realizó junto a Du Potet experimentos y demostraciones de mesmerismo ${ }^{88}$. Después de un rápido éxito, Elliotson fue acusado de fraude y tuvo que dejar su puesto académico. Más tarde fundó un hospital de tratamientos mesmericos (London Mesmeric Infirmary) y la revista The Zoist (1843-1856) ${ }^{89}$. Townshend, amigo íntimo de Elliotson, también realizó sus experimentos mesmericos entre 1838 y 1839 en el Trinity College de Cambridge ${ }^{90}$. En síntesis, el magnetismo animal fue expulsado de los espacios académicos de Inglaterra pero siguió fascinando durante mucho tiempo a escritores e intelectuales, entre ellos Doyle y Du Maurier.

La novela Trilby, de Du Maurier, es considerada como el primer bestseller de la era moderna. Publicada por entregas en Harper's New Monthly Magazine entre enero y agosto de 1894, el relato tuvo éxito inmediato, llegándose incluso a hablar de una «Trilbymanía». La novela fue publicada en formato de libro, alcanzando siete ediciones antes de terminar $1894^{91}$. La venta de la novela a los editores norteamericanos HarperEBrothers y de los derechos de la adaptación teatral convirtieron a su autor en millonario. Du Maurier pasó su vida entre Londres y París (su madre era inglesa y su padre francés), estudió pintura en París pero una lesión en un ojo le impidió dedicarse a ella. En Londres, trabajó como ilustrador y caricaturista para la revista Punch. Su primera novela Peter Ibbetson (1891) la publicó con 57 años. Tres años después se publicó Trilby, y dos años más tarde, en 1896, falleció. Su tercera y última novela The Martian (1896) se publicó póstumamente $^{92}$. Según Philip Holden, es difícil encontrar una novela del

\footnotetext{
86. Gauld, n. 5, p. 203-205.

87. Fara, Patricia. Fatal attraction: Magnetic mysteries of the Enlightenment. Cambridge: Icon Books; 2005.

88. Winter, n. 74, p. 60-78.

89. El título completo de la revista es The Zoist. A Journal of Cerebral Physiology and Mesmerism, and their Applications to Human Welfare y se considera una de las más importantes fuentes de información sobre el mesmerismo británico de ese periodo. Gauld, n. 5, p. 219-234.

90. Winter, n. 74, p. 31-59.

91. Purcell, Edward. Trilby and Trilby-Mania: The beginning of the bestseller system. Journal of Popular Culture. 1977; 11: 62-76.

92. Petroski, Nicole. Double agency in George Du Maurier's novel Trilby. Regina: University of Regina; 1997.
} 
período victoriano tardío en la que no aparezcan la hipnosis, la posesión, el mesmerismo o los médiums ${ }^{93}$. En este sentido, Du Maurier fue el prototipo de escritor victoriano ya que sus tres novelas exploran diferentes aspectos del mundo de las experiencias extrasensoriales ${ }^{94}$. Mientras en Peter Ibbetson Du Maurier se centra en los sueños lúcidos y los viajes del alma fuera del cuerpo, en Trilby explora la dominación del otro mediante el magnetismo animal y en The Martian presenta el tema de las reencarnaciones infinitas ${ }^{95}$.

Trilby ha servido de fuente para trabajos académicos centrados en la imagen de la mujer, el antisemitismo, la eugenesia racial, el darwinismo, la imagen de las clases sociales y el mesmerismo ${ }^{96}$. En la novela, ambientada en el París de mediados del siglo XIX, una chica llamada Trilby sin ningún oído musical, lavandera y musa de diferentes artistas bohemios del lugar, se convierte, años después, en la mejor cantante lírica del mundo. El éxito de Trilby como cantante es debido a la oculta dominación e influencia mesmerica de un maestro de música, el terrible y odioso Svengali, que también ha aprovechado su poder para conseguir casarse con ella. Trilby no es consciente de que está bajo el poder mesmerico de Svengali. Tras la trágica muerte de éste, consigue liberarse de la influencia mesmerica, cae enferma y muere poco tiempo después.

En referencia al gran cambio que se producía en Trilby en estado mesmerico, el ayudante de Svengali nos dice: «Había dos Trilbys» ${ }^{97}$ y «Svengali la convertía en otra Trilby» ${ }^{98}$. John Elliotson afirmaba que podía tocar, como si de un piano se tratase, el cerebro de alguien mesmerizado previamente por él y conseguir grandes cambios en las habilidades de esa persona ${ }^{99}$. Algo similar relató Puysegur sobre Victor Race, un campesino iletrado que en estado de sonambulismo hablaba en un lenguaje muy refinado ${ }^{100}$. Sin embargo, el parecido más exacto con la historia de Trilby lo encontramos

93. Holden, Philip. Castle, coffin, stomach: Dracula and the banality of the occult. Victorian Literature and Culture. 2001; 29 (2): 469-485.

94. Existe una traducción al español de su primera novela: Du Maurier, George. Peter Ibbetson. Madrid: Aguilar; 1962. Por el contrario, no hay traducción española de The Martian.

95. Stevenson, Lionel. George Du Maurier and the Romantic novel. In: Wallis, Hardy, ed. Essays by Divers Hands. London: Oxford University Press; 1960, p. 3 6-54.

96. Vorachek, Laura. Mesmerist and other meddlers: Social Darwinism, degeneration, and eugenics in Trilby. Victorian literature and culture. 2009; 37: 197-215.

97. Du Maurier, n. 11, p. 427

98. Du Maurier, n. 11, p. 427.

99. Winter, n. 74, p. 63.

100. Peter, n. 39, p. 39-40. 
en un experimento realizado por James Braid. Éste consistió en hacer cantar con extremo virtuosismo a una joven hipnotizada que nada sabía de canto o música y que fue presenciado en su papel de experta por Jenny Lind, la más afamada cantante lírica de la época ${ }^{101}$. La anterior anécdota ha servido como fuente de especulación para algunos autores que han señalado como modelo de Svengali y Trilby a Braid y Lind, respectivamente ${ }^{102}$. Los grandes cambios de personalidad descritos, frecuentes en las personas en estado de mediumnidad (casi siempre mujeres) y explicados desde las teorías espiritistas por la conexión del médium con los espíritus, fueron el origen de las primeras teorías psiquiátricas sobre el desdoblamiento de la personalidad o personalidad múltiple ${ }^{103}$.

El personaje de Svengali es presentado en la novela como de «aspecto siniestro» ${ }^{104}$, «su vanidad y soberbia resultaban insoportables. Además era pretencioso y sucio en su manera de vestir. No era un hombre simpático» ${ }^{105}$, «recorriendo el mundo en busca de alguien a quien engañar, traicionar, explotar, de quien burlarse brutalmente» ${ }^{106}$, siempre dispuesto a «mortificar, asustar, tiranizar o atormentar a cualquiera» ${ }^{107}$. Además, se usa el hecho de que Svengali es judío para profundizar en la imagen de degeneración y maldad del personaje: «Svengali era un judío oriental y no pudo resistirse a la tentación de escupirle en la cara» ${ }^{108}$. La novela está cargada de fuertes alusiones al carácter judío de Svengali, lo que la ha convertido en un icono del antisemitismo de la época ${ }^{109}$. Recordemos que el famoso caso de Dreyfus se hizo público en el mismo año de la publicación de Trilby, algo que sin duda influyó en Du Maurier ${ }^{110}$.

101. Braid, James; Robertson, Donald, ed. The discovery of hypnosis: The complete writings of James Braid. Bilston: Nch Ltd; 2009.

102. Grimes, Hilary. Identities and powers in flux: Mesmerism, hypnotism, and George Du Maurier's Trilby. In: Grimes, n. 69, p. 61-81.

103. Plas, Régine. Naissance d'une science humaine: la psychologie. Les psychologues et le merveilleux psychique. Rennes: Presses Universitaires de Rennes; 2000.

104. Du Maurier, n. 11, p. 20

105. Du Maurier, n. 11, p. 63.

106. Du Maurier, n. 11, p. 65

107. Du Maurier, n. 11, p. 114.

108. Du Maurier, n. 11, p. 384.

109. Rosenberg, Edgar. From Shylock to Svengali: Jewish stereotypes in English fiction. Stanford: Stanford University Press; 1960.

110. Davidson, Neil. The Jew as homme/femme-fatale: Jewish (Art)ifice, Trilby, and Dreyfus. Jewish Social Studies. 2002; 8 (2/3): 73-111. 
El método mesmerico de Svengali comienza por mirar fijamente al blanco de los ojos y «darle pases magnéticos sobre la frente y las sienes, sobre el cuello y la garganta» ${ }^{111}$, ordenándole «ahora duérmete» ${ }^{112}$. Trilby, una vez en estado normal, sin el influjo del mesmerismo de Svengali, presentaba una amnesia para todo lo ocurrido en estado mesmerico, incluso para periodos de meses y años ${ }^{113}$.

Como fuente de inspiración para el personaje de Svengali se ha señalado a Grigori Rasputin (1869-1916). Resulta innegable el parecido de los dibujos de Svengali realizados por Du Maurier con las fotografías de Rasputin ${ }^{114}$. Otros modelos sugeridos incluyen desde pianistas del entorno de amistades del propio Du Maurier, como el griego Sotiri y Henri Litolff, a músicos virtuosos como Paganini, Chopin y Schubert ${ }^{115}$. Winter ha apuntado la similitud de los gestos y de las expresiones faciales del director musical y del magnetizador ${ }^{116}$.

El modelo más plausible para Svengali fue Felix Moscheles (18331917), íntimo amigo de Du Maurier ${ }^{117}$. Moscheles, que también era judío, fue un estudioso del magnetismo animal, además de pintor, pianista y escritor inglés. Conoció a Du Maurier durante su época de estudiante en Amberes, donde fueron compañeros de habitación ${ }^{118}$. Las aventuras de los dos amigos en París están retratadas por el propio Moscheles en el libro In Bohemia with Du Maurier. The First of a Series of Reminiscences (1896) ${ }^{119}$. En él, Moscheles confiesa que «Mesmerism, or, as the fashion of today calls it, Hypnotism, formed so frequent a topic of conversation and speculation between Du Maurier and myself» ${ }^{120}$. Algún tiempo antes de la publicación de

111. Du Maurier, n. 11, p. 74

112. Du Maurier, n. 11, p. 370.

113. Du Maurier, n. 11, p. 377

114. Pick, Daniel. Svengali's Web: The alien enchanter in Modern culture. Suffolk: St. Edmundsbury Press; 2000.

115. Ormond, Leonée. George Du Maurier. London: Routledge \& Kegan Paul; 1969.

116. Winter, n. 74, p. 310-314.

117. Petroski, n. 93, p. 100.

118. Davidson, n. 110, p. 83.

119. Moscheles, Felix. In Bohemia with Du Maurier. The first of a series of reminiscences. With 63 original drawings by G. Du Maurier, illustrating the artist's life in the fifties. London: T. F. Unwin; 1896.

120. Moscheles, n. 119, p. 51. 
Trilby, Du Maurier afirmaba en una carta dirigida a Moscheles: «You'll see that I've used up all your Mesmerism and a trifle more in my new book» ${ }^{121}$.

Tras la muerte de Du Maurier en 1896, la Trilbymanía se fue apagando y la novela Trilby fue poco a poco olvidándose. Sin embargo, la figura de Svengali llegó a ser un icono popular de la sociedad victoriana de fin-de-siècle.

\section{Conclusiones}

El análisis de la imagen del magnetismo animal en la literatura de ficción puede ser muy útil e interesante recorriendo dos vías paralelas, pero no necesariamente excluyentes, apuntadas en la introducción. Por un lado, comprender mejor las fuentes y la génesis del estereotipo del mesmerizador como alguien malvado, amoral y peligroso, y de la representación del magnetismo animal y de la hipnosis como pseudociencias esotéricas que proporcionan un poder ilimitado sobre el otro, además de las más increíbles facultades paranormales como regresiones a vidas pasadas, telepatía, clarividencia, etc. Este intento de comprender los mitos actuales de la hipnosis acercándose al pasado mediante unos textos novelados, cuyas líneas argumentales descansan en el mesmerismo o hipnosis, sin duda ya es partidista, situándose al lado de las reales comisiones científicas parisinas que examinaron las prácticas mesmericas para intentar separar el trigo de la paja. Desde entonces hasta la actualidad, muchos han sido los que han intentado «desmitificar la hipnosis». Por otro lado, tenemos en la literatura de ficción una fuente única e inestimable de datos, ideas, especulaciones, preocupaciones y posibilidades en torno al magnetismo animal e hipnosis que convierten su estudio y análisis en un capítulo imprescindible de cualquier trabajo histórico de este tema. Debemos tener en cuenta que la labor para desprestigiar al magnetismo animal comenzó muy pronto, con las reales comisiones mencionadas. Más adelante se introdujo la hipnosis dentro de la ciencia médica ortodoxa, aunque marginalmente. El magnetismo animal, para ser aceptado por la academia en sentido general, tuvo que ser filtrado por los parámetros cientificistas de la época y reducido a estados psíquicos patológicos o a sugestiones psicológicas ${ }^{122}$. Lo anterior conlleva

121. Moscheles, n. 119, p. 9.

122. Montiel; González de Pablo, n. 5, p. 28-31. 
un ocultamiento, un olvido y un menosprecio del magnetismo animal en sentido amplio del término, por lo que el estudio de la literatura de ficción sobre este tema puede proporcionar hallazgos históricos que difícilmente encontraremos en otro lugar.

Hemos visto como el uso literario del mesmerismo en el caso de Poe, Doyle y Du Maurier no es algo casual o periférico, sino que estos autores estuvieron intelectualmente interesados y estimulados por estas ideas. En este sentido, el mesmerismo forma parte importante de sus biografías y del contexto de la creación de sus relatos. Sin embargo, podemos observar diferencias en el tratamiento del tema según los autores, desde el atrevimiento a cruzar límites morales y la curiosidad científica de los magnetizadores de Poe y Doyle hasta el malvado y diabólico Svengali de Du Maurier. Sin entrar en consideraciones literarias y refiriéndonos en exclusiva al tema del mesmerismo, podríamos decir que Poe y Doyle comparten y coinciden mucho más en un mismo background mesmerico que los diferencia de $\mathrm{Du}$ Maurier, y a su vez este último, refleja en su relato mucho más el estereotipo del hipnotizador villano. 